\title{
Proteomic Analysis of High Temperature Stress-Responsive Proteins in Chrysanthemum Leaves
}

\author{
Xin Li ${ }^{\#}$, Feiya Liao", Qiqi Ma, Beibei Jiang , Yuanzhi Pan, Cheng Luo, Xinjie Wang and Aining Ran \\ Department of Ornamental Horticulture, Sichuan Agricultural University, Chengdu, 611130, China \\ ${ }^{*}$ Corresponding Author: Beibei Jiang. Email: 13786@sicau.edu.cn \\ \#These authors contributed equally to this work
}

Received: 17 February 2021 Accepted: 29 March 2021

\begin{abstract}
Chrysanthemum is one of the most important ornamental flowers in the world, and temperature has a significant influence on its field production. In the present study, differentially expressed proteins were investigated in the leaves of Dendranthema grandiflorum 'Jinba' under high temperature stress using label-free quantitative proteomics techniques. The expressed proteins were comparatively identified and analyzed. A total of 1,463 heat-related, differentially expressed proteins were successfully identified by Liquid Chromatography-tandem Mass Spectrometry (LC-MS/MS), and 1,463 heat-related, differentially expressed proteins were successfully identified by mass spectrometry after a high temperature treatment. Among these, 701 proteins were upregulated and 762 proteins were downregulated. The in-depth bioinformatics analysis of these differentially expressed proteins revealed that these were involved in energy metabolism pathways, protein metabolism, and heat shock. In the present study, the investigators determined the changes in the levels of some proteins, and their expression at the protein and molecular levels in chrysanthemum to help reveal the mechanism of heat resistance in chrysanthemum. Furthermore, the present study elucidated some of the proteins correlated to heat resistance in chrysanthemum, and their expression changes at the protein and molecular levels to help reveal the mechanism of heat resistance in this flower species. These results provide a theoretical basis for the selection of new heat resistant varieties of chrysanthemum in the field.
\end{abstract}

\section{KEYWORDS}

Chrysanthemum; high temperature stress; proteomics; differential protein; expression

\section{Introduction}

Chrysanthemum (Dendranthema morifolium [Ramat.] Tzvel.) is a perennial flower that has been cultivated in China for more than 3,000 years due to its variable flower shape, noble meaning, and certain medicinal value. It is popular among consumers, and has significant economic and social benefits [1].

The field production of chrysanthemum is influenced by many factors. Among these, temperature is one of them. The optimum temperature for the growth and development of chrysanthemum is $15-25^{\circ} \mathrm{C}$. Temperatures above $25^{\circ} \mathrm{C}$ are unfavorable for its growth, which basically stops at $40^{\circ} \mathrm{C}$ [2]. However, due to the trend of global warming in recent years, most parts of the China have experienced hot summers, and extremely high temperatures above $40^{\circ} \mathrm{C}$. Hence, high temperature heat damage has 
become one of the important factors that affected chrysanthemum production [3]. Dendranthema grandiflorum 'Jinba' is a popular chrysanthemum variety in the market. This is a heat resistant chrysanthemum variety that can withstand a maximum temperatures of $32^{\circ} \mathrm{C}$. Physiological studies have found that after a high temperature treatment, the change in electrical conductivity was small, and malondialdehyde (MDA) increased with the number of days of heat stress. Hence, it was concluded that Dendranthema grandiflorum 'Jinba' was the more heat resistant variety [4].

When plants are exposed to high temperatures, temperature changes stimulate a stress response, which in turn triggers changes in protein expression in plants. These changes induce the synthesis of proteins related to the plant heat resistance mechanism to improve plant heat resistance. However, protein and molecular level studies related to the heat resistance of Dendranthema grandiflorum 'Jinba' have not been conducted. Conducting these studies could help to clarify the mechanism of the high temperature stress in chrysanthemum.

In the present study, a quantitative proteomic study was carried out on chrysanthemum treated either at room or high temperature using non-labeled quantitative proteomic techniques. Then, the differentially expressed proteins induced by high temperature were screened and identified, and subsequently analyzed by bioinformatics.

\section{Materials and Methods}

\subsection{Plant Materials}

The Dendranthema grandiflorum 'Jinba' used for the present study was planted in Sichuan Agricultural University. In the tissue culture room, routine maintenance was carried out according to the tissue culture conditions and specific needs. The plants were transplanted into $7 \mathrm{~cm} \times 7 \mathrm{~cm} \times 13 \mathrm{~cm}$ plastic pots, with one plant per pot, according to the conventional cultivation method. Basic seedlings underwent routine management.

\subsection{High Temperature Processing and Leaf Collection}

Potted plants with good growth and ten-leaf age were selected and transferred to an artificial climate chamber set at either $25^{\circ} \mathrm{C}$ (control group) or $40^{\circ} \mathrm{C}$ (experimental group) for three days. All other treatment conditions were kept the same. The photoperiod was $16 \mathrm{~h}$ light and $8 \mathrm{~h}$ dark, with $70 \%$ relative humidity. At the end of the high-temperature treatment, the fourth and fifth leaves from the apical part of each plant were collected, mixed and sampled to ensure three biological replicates. Then, these were frozen in liquid nitrogen, and stored at $-80^{\circ} \mathrm{C}$ in a refrigerator for later use.

\subsection{Protein Extraction}

The sample was grinded by liquid nitrogen into cell powder, and transferred to a $5 \mathrm{~mL}$ centrifuge tube. Afterwards, four volumes of lysis buffer ( $8 \mathrm{M}$ of urea, $1 \%$ protease inhibitor) were added to the cell powder, followed by sonication for three times on ice using a high intensity ultrasonic processor (Scientz) (Note: For the post-translational modification [PTM] experiments, inhibitors were also added to the lysis buffer; that is, $3 \mu \mathrm{M}$ of trichostatin A [TSA] and $50 \mathrm{mM}$ of $\mathrm{N}$-acetylmuramic acid [NAM] for acetylation). The remaining debris was removed by centrifugation at $12,000 \mathrm{~g}$ at $4^{\circ} \mathrm{C}$ for $10 \mathrm{~min}$. Finally, the supernatant was collected, and the protein concentration was determined using a BCA kit, according to manufacturer's instructions.

\subsection{Trypsin Digestion}

For digestion, the protein solution was reduced with $5 \mathrm{mM}$ of dithiothreitol for $30 \mathrm{~min}$ at $56^{\circ} \mathrm{C}$, and alkylated with $11 \mathrm{mM}$ of iodoacetamide for $15 \mathrm{~min}$ at room temperature in darkness. Then, the protein sample was diluted by adding $100 \mathrm{mM}$ of tetraethylammonium bromide (TEAB) to the urea 
concentration at less than $2 \mathrm{M}$. Finally, trypsin was added at a 1:50 trypsin-to-protein mass ratio for the first digestion overnight, and at a 1:100 trypsin-to-protein mass ratio for a second four-hour digestion.

\subsection{LC-MS/MS Analysis}

The tryptic peptides were dissolved in $0.1 \%$ formic acid (solvent A), and directly loaded onto a homemade reversed-phase analytical column $(15 \mathrm{~cm}$ in length, $75 \mu \mathrm{m}$ id). Then, the gradient comprised of an increase from $6 \%$ to $23 \%$ solvent B $(0.1 \%$ formic acid in $98 \%$ acetonitrile $)$ over 26 min, an increase from $23 \%$ to $35 \%$ in eight minutes, and an increasing trend to $80 \%$ in three minutes. This was followed by holding at $80 \%$ for the last three minutes, and all were maintained at a constant flow rate of $400 \mathrm{~nL} / \mathrm{min}$ on the EASY-nLC 1000 Ultra Performance Liquid Chromatography (UPLC) system.

The peptides were subjected to the NSI source, followed by tandem mass spectrometry (MS/MS) in Q Exactive TM Plus (Thermo) coupled online to the UPLC. The electrospray voltage applied was $2.0 \mathrm{kV}$. The $\mathrm{m} / \mathrm{z}$ scan range was 350-1,800 for the full scan, and intact peptides were detected in the Orbitrap at a resolution of 70,000. Then, the peptides were selected for MS/MS using the Normalized Collisional Energy (NCE) set at 28, and the fragments were detected in the Orbitrap at a resolution of 17,500. The data-dependent procedure alternated between MS scans, followed by $20 \mathrm{MS} / \mathrm{MS}$ scans with $15.0 \mathrm{~s}$ of dynamic exclusion. Automatic gain control (AGC) was set at 5E4. The fixed first mass was set at $100 \mathrm{~m} / \mathrm{z}$.

\subsection{Database Searches}

The resulting MS/MS data were processed using the MaxQuant search engine (v.1.5.2.8). Tandem mass spectra were searched against the human uniprot database concatenated with the reverse decoy database. Trypsin/P was specified as the cleavage enzyme, allowing up to four missing cleavages. The mass tolerance for precursor ions was set at $20 \mathrm{ppm}$ in the first search, and $5 \mathrm{ppm}$ in the main search; the mass tolerance for fragment ions was set at $0.02 \mathrm{Da}$. The Carbamidomethyl on Cystine (Cys) was specified as fixed modification and acetylation modification and oxidation on Met were specified as variable modifications. False discovery rate (FDR) was adjusted to $<1 \%$, and the minimum score for modified peptides was set at $>40$.

\subsection{Bioinformatics Analysis of the Identified Proteins}

The relative quantification values for each sample were calculated based on the Label-free Quantification (LFQ) intensity of the protein between the different samples. Then, the $P$-value was calculated using the two-sample, two-tailed t-test method. Except for the $P$-value of $<0.05$, the A 1.5 -fold change was set to identify the significantly differentially expressed proteins.

The Gene Ontology (GO) identity of each of the identified proteins was obtained in the InterProScan search. The GO classification of these proteins was performed using UniProt. The Gene Ontology Annotation (GOA) database and annotated data for identifying proteins were obtained.

\section{Results}

\subsection{Protein Identification and Quantification}

Non-labeled quantitative proteomics was applied to six samples (H1, H2, H3, CK1, CK2 and CK3) for mass spectrometry, and this identified 38,352 peptides (31,914 specific peptides) and 6,313 proteins. Among these, 4,513 were quantifiable (quantitative information available for at least one comparison group). Statistical analysis of the software revealed (Fig. 1) that there were 1,463 significant differential protein spots. Among these, 701 were upregulated and 762 were downregulated. 


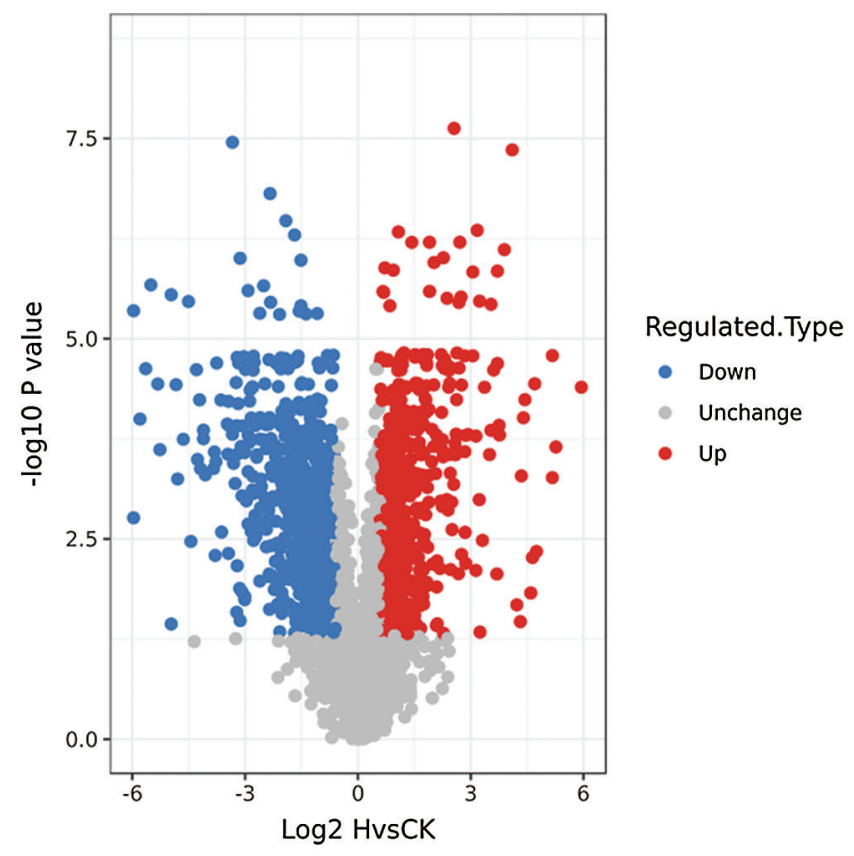

Figure 1: The HvsCK volcano plot. The horizontal axis is the relative quantification value of the protein after $\log 2$ conversion, and the vertical axis is the $P$-value of the significance test after the $-\log 10 \operatorname{logarithmic}$ transformation. The red dots indicate the significantly and differentially expressed upregulated proteins, and the blue dots indicate the significantly and differentially expressed downregulated proteins

\subsection{Functional Analysis of Differentially Expressed Proteins}

A total of 1,463 differential proteins were successfully identified and analyzed using the MaxQuant software. The identified proteins were divided into 22 groups, based on their putative functions, in order to further elucidate the mechanism of heat resistance on chrysanthemum leaves.

The Cluster of Orthologous Groups of proteins (COG) classification of the differential proteins is shown in Fig. 2, where 476 related proteins were involved in energy metabolism, and accounted for $41 \%$ of the total identified proteins. Furthermore, 57 associated proteins were involved in the construction of cellular structures, accounting for $5 \%$ of the total number of proteins identified. Also, 57 associated proteins were involved in chaperones, in which 224 were associated proteins, accounting for $20 \%$ of the total proteins identified. Three hundred and sixteen associated proteins were involved in other functions, accounting for $27 \%$ of the total number of proteins identified. Finally, 80 unknown proteins accounted for $7 \%$ of the total number of proteins identified. In terms of the proportion of differentially expressed proteins in functional categories, the greatest differences were found in the expression of the energy metabolism and molecular chaperone-related proteins under heat stress.

We investigated and screened the top 50 most closely interacting proteins, and used the to map the protein interaction network (Fig. 3). This was to gain a comprehensive understanding of the differentially expressed proteins affected by heat stress, and clearly demonstrate the protein-protein interactions. The size of the circles represents the number of differential proteins and their interacting proteins. The larger the circle, the more proteins it interacted with, and the more important the protein was in the network.

The upregulated proteins (spot 984, 1400) were found to play a key role in the network, as shown in Fig. 3. The data analysis revealed that this was separately associated with the defensive responses, and that energy metabolism and biosynthetic processes were correlated. Furthermore, it was speculated that 
these may be involved in the process of high-temperature stress in chrysanthemum. The downregulated proteins (spot 42, 1141), which play a key role in the network, were found to be mainly associated with the energy metabolism and biosynthesis processes. Therefore, the results of the present study indicated that the energy metabolism and biosynthesis were the main biological processes involved in the hightemperature stress of Dendranthema grandiflorum 'Jinba'.

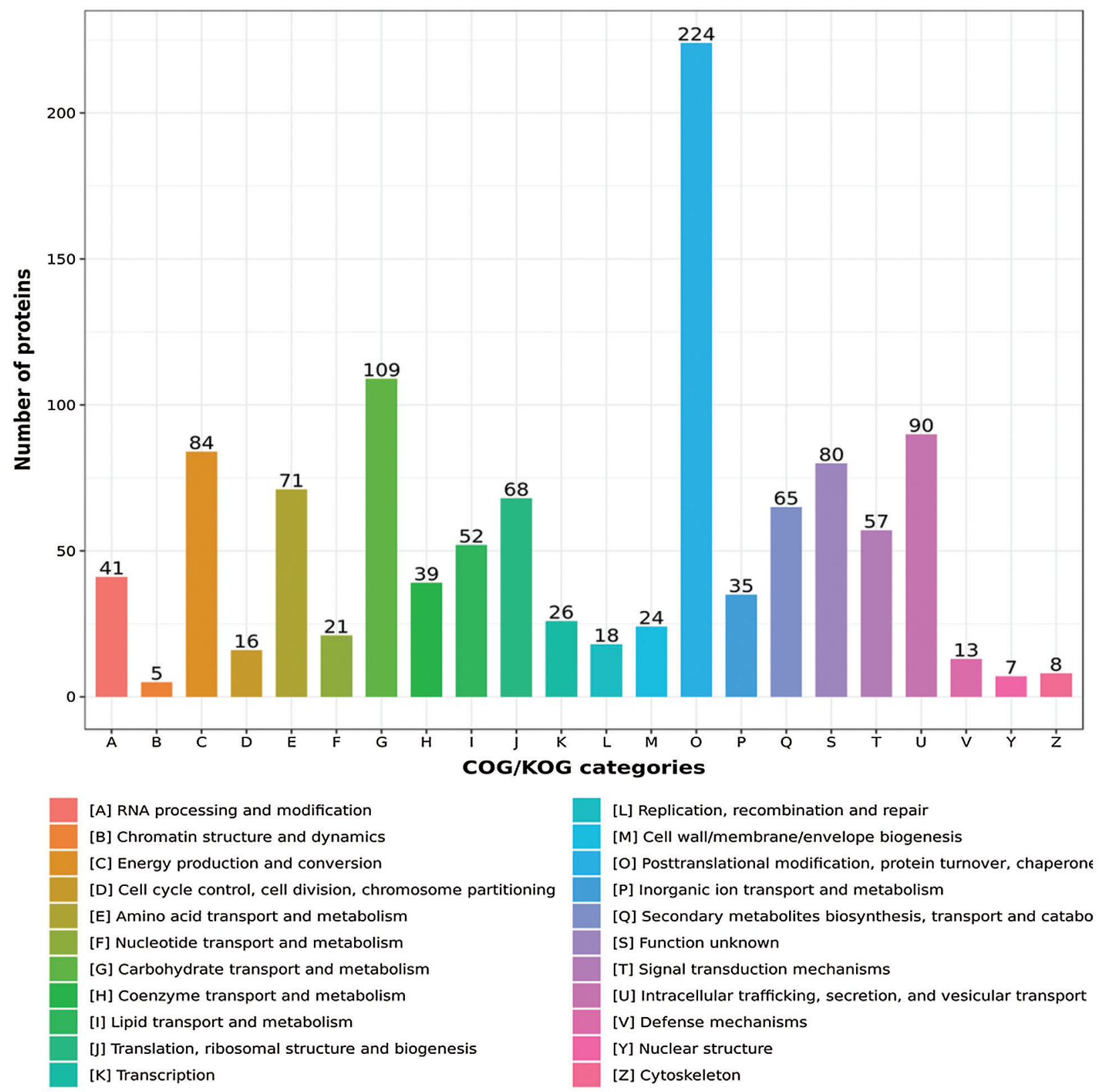

Figure 2: $\mathrm{COG} / \mathrm{KOG}$ categorie. The horizontal axis is the letter number of the COG classification, and the correspondence between letters and classification is fixed 


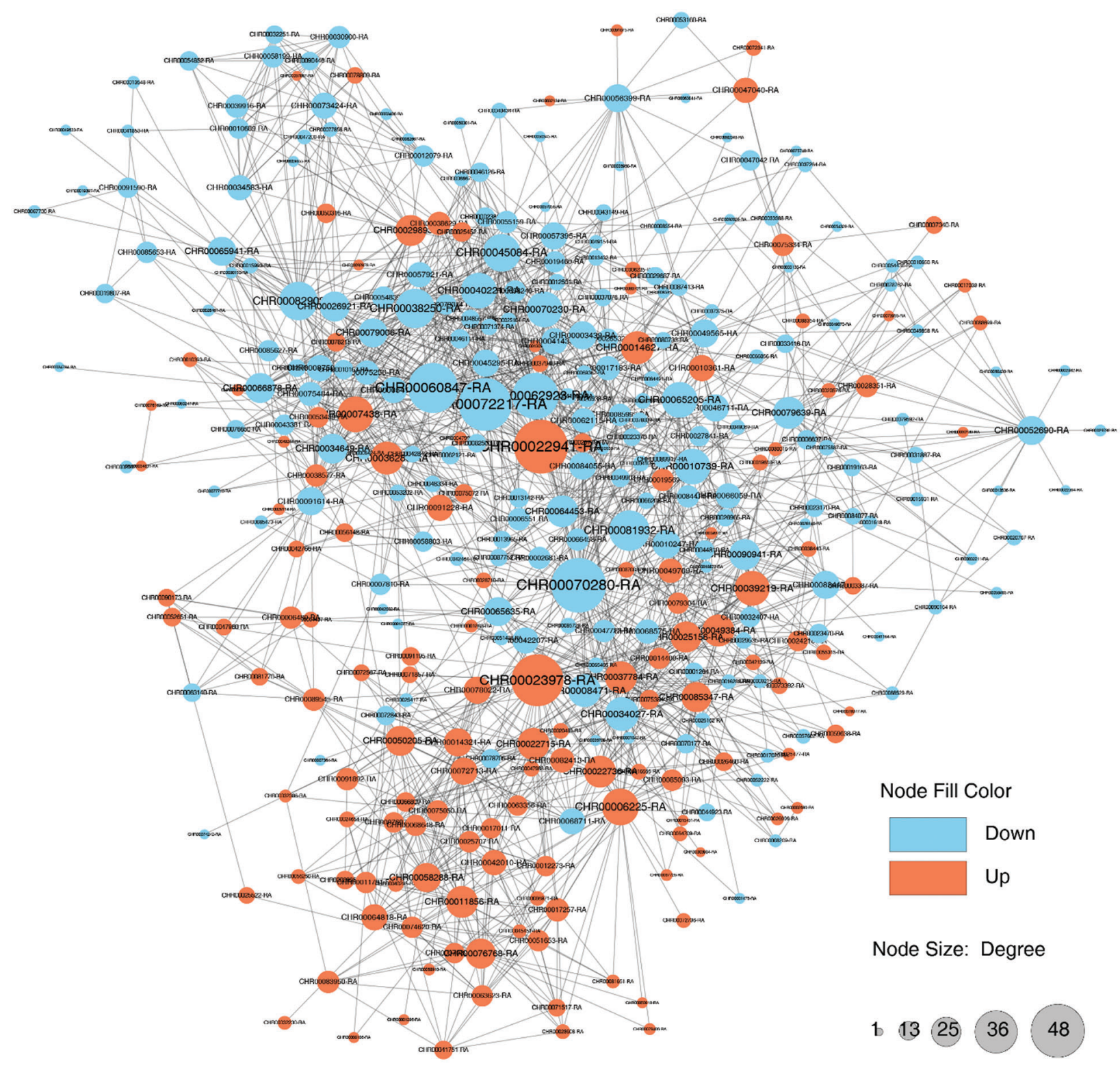

Figure 3: Differential proteins interaction network. The circles represent differentially expressed proteins, and the colors represent the differential expression of the proteins (blue for down-regulated proteins, red for up-regulated proteins). The size of the circle represents the number of proteins (degree) that the different proteins interacted with. The larger the circle, the more proteins it interacted with, and the more important the protein was in the network

\section{Discussion}

With the rapid development of plant proteomics, the use of proteomic technology has been isolated from a variety of ornamental plants, such as moonflower and lily. It identifies a series of high-temperature stress, response-related proteins. It was found that high-temperature stress can affect resistance-related proteins, such as heat stress proteins, energy and changes in the expression of proteins related to substance metabolism [5]. However, proteomic studies on the heat resistance of Dendranthema grandiflorum 'Jinba' were lacking. 
The present study elucidated the molecular mechanisms of heat stress in Dendranthema grandiflorum 'Jinba' through the proteomic analysis by mass spectrometry. Many changes were observed at the protein level of energy and metabolic pathways, chaperones, and other functional proteins. The results of the proteomic analysis are discussed in detail below.

\subsection{Energy Metabolizing Protein}

Maintaining a basic energy supply is essential for plants to resist adversity. Therefore, plants can respond to adversity stresses by regulating their energy metabolism [6]. The proteomic analysis of rice under heat stress conducted by Lin et al. [7] revealed that the mechanism of the response to heat stress is complex, and that the differential proteins include many proteins associated with different metabolic pathways, which are both upregulated and downregulated. In the present study, the proteins identified were associated with photosynthesis, and their expression levels were downregulated. Furthermore, some proteins were related to the energy metabolism pathway, and their expression levels were either upregulated or downregulated at least at some point in time. This also indicated that heat stress can severely affect energy metabolic pathways.

Hexose kinase is the key enzyme that catalyzes glycolysis, while fructose kinase is a type of hexose kinase that catalyzes fructose in the basic metabolism of plant phosphorylation, thereby affecting the process of glycolysis [8]. In the study conducted by Wang et al. [9] on rice spikelet inflorescence, it was revealed that the expression of fructose kinase is upregulated under heat stress. In the present study, fructose kinase exhibited a downregulated expression in response to heat stress, indicating that fructose kinase expression is complex, and more research is needed to prove this. The study conducted by Li et al. [10] revealed that in tomato, the overexpression of the hexose kinase would affect plant growth and photosynthesis, leading to a severe and rapid senescence of photosynthetic tissues.

These results are consistent with the work of Wang, who reported that cellular glycolysis during warming from $30^{\circ} \mathrm{C}$ to $38^{\circ} \mathrm{C}$ was the main pathway for metabolic regulation [11]. This confirms that fructose, as a plant hexose receptor and signaling molecule, can regulate metabolism and growth by influencing the plant life cycle [12].

\subsection{Chaperone}

Heat-shock proteins (HSPs) act as molecular chaperones that bind to denatured proteins to form aggregates and reduce the damage caused by the adversity on plants. In plant leaves, these chaperones protect important organelles, such as photosynthetic systems and mitochondria, from damage under adverse conditions $[13,14]$.

It has been demonstrated that HSP22 is involved in the folding of newly synthesized proteins in response to cellular stress, maintaining their proper configuration, allowing transmembrane proteins to cross the organism, aiding the transmembrane transport, and facilitating the recovery of damaged and denatured proteins [15]. Li's study on soybean seedlings treated with high-temperature stress revealed that differentially expressed proteins present in the treated leaves, stems and roots were mostly associated with functions such as heat-stress proteins, photosynthesis and protein synthesis [16]. Shi et al. [17] reported a number of differentially expressed proteins in maize seedling leaves after high-temperature stress, with the upregulated expression mainly for heat-stress proteins, and glycolysis- and photosynthesis-related proteins; the downregulated expression was mainly for protein synthesis-related proteins and secondary metabolism-related proteins.

The upregulated differentially expressed proteins found in leaves under high-temperature stress in the present study were mainly heat-stress proteins, photosynthesis-related proteins, etc. The downregulated expression was mainly for protein synthesis and secondary metabolism-related proteins. This is similar to 
some of the results of the previous studies mentioned above. Hence, it can be concluded that heat-stress proteins play an important role in plant heat tolerance, and that the heat-shock in the upregulation of proteins implies that protein folding and assembly are enhanced by high temperature.

In addition, the present study also identified a small-molecule heat-excited protein of the HSP20 family by identification (spot 123, 290). Ul Haq et al. [18] reported that the accumulation of HSP20 was closely correlated to the acquisition of heat resistance. In addition, Muthusamy et al. [19] reported that its HSP accumulation and overexpression could also improve the heat resistance of crops. In the present study, the high temperature treatment induced the significant upregulation of the HSP20 expression, which was presumed to be involved in the folding of heat stress-associated proteins in chrysanthemum and assembly, aiding the transmembrane transport, and facilitating the recovery of damaged and denatured proteins.

\subsection{Other Functional Proteins}

In the present study, proteins associated with protein synthesis were found to be upregulated in leaves under high temperature, and eukaryotic translation initiation factor 3 was upregulated. Eukaryotic translation initiation factor 3 subunit J-A-like and eukaryotic translation initiation factor 6-2 downregulated the expression of eukaryotic translation initiation factor 4B3-like and chloroplast translation initiation factor IF-2. These results imply that the balance of protein metabolism is sharply adjusted in leaves affected by high temperature, which may be due to the fact that leaves have more functional proteins damaged by heat. Therefore, these require more protein synthesis for self-replenishment, suggesting that anabolic metabolism and the transport of proteins were necessary to counteract heat stress.

\section{Conclusion}

A differential proteomic approach was applied to analyze the differential proteins in the leaves of Dendranthema grandiflorum 'Jinba' after high-temperature treatment, and 6,313 were identified by mapping analysis. Among these, 4,513 were quantifiable, and a total of 1,463 significantly differentially expressed proteins were obtained by non-labeled quantification methods. Among these, 701 differentially expressed proteins were upregulated and 762 differentially expressed proteins were downregulated. The relevant differentially expressed proteins were involved in energy and metabolic pathways, protein metabolism, chaperones, etc. Furthermore, HSP20 played an important role in the heat-resistant properties of Dendranthema grandiflorum 'Jinba', but its action mechanism remains unclear. The subsequent functional validation of these differential proteins would be helpful for the study of the heat-resistant mechanisms in chrysanthemum, and the selection of new heat-resistant varieties.

Acknowledgement: The authors thank Hangzhou Jingjie Biotechnology Co., for the technology support.

Funding Statement: This research was supported by the National Natural Science Foundation of China (31800601) and Innovation Training Program for College Students of Sichuan Agricultural University (040-2021998185).

Conflicts of Interest: The authors declare that they have no conflicts of interest to report regarding the present study.

\section{References}

1. Zhang, H. (2017). Expression of fatty acid desaturase gene and changes in antioxidant enzyme activity in chrysanthemum under high temperature stress (Master's Thesis). Henan Agricultural University.

2. Kong, L., Chen, Y., Wang, Y. (2019). Heat tolerance of different summer chrysanthemum varieties. Acta Horticulturae Sinica, 46(12), 2437-2448. DOI 10.16420/j.issn.0513-353x.2019-0145. 
3. Sagheer, A. (2019). Study on the mechanism of high temperature inhibiting axillary bud growth of chrysanthemum (Ph.D. Thesis). Beijing Forestry University.

4. Fu, K. (2017). Tolerance of three chrysanthemum varieties to high-temperature stress. Shanghai Agricultural Science and Technology, (3), 83-85. DOI 10.3969/j.issn.1001-0106.2017.03.049.

5. Kumar, N., Suyal, D. C., Sharma, I. P., Verma, A., Singh, H. (2017). Elucidating stress proteins in rice (Oryza sativa L.) Genotype under elevated temperature: A proteomic approach to understand heat stress response. 3 Biotech, 7(3), 205. DOI 10.1007/s13205-017-0856-9.

6. He, L. X. Z., Jia, Z. Q., Liu, T., Li, Q. X., Zhang, Y. Y. et al. (2018). Research progress of plant adaptability towards adversity stress. World Forestry Research, 31(2), 13-18. DOI 10.13348/j.cnki.sjlyyj.2018.0003.y.

7. Lin, C. X., Ni, D. H., Song, F. S., Hu, Z. B., Gan, Q. (2020). Research progress on physiological characteristics and adaptation mechanism of rice under high temperature stress. Anhui Agricultural Science Bulletin, $26(24), 37-42$. DOI 10.16377/j.cnki.issn1007-7731.2020.24.012.

8. Wang, Y. J., Zhang, S. J., Lai, Y., Hu, Y. F. (2019). Identification and expression analysis of genes encoding carbohydrate metabolism enzymes and sugar transporters. Hubei Agricultural Sciences, 58(22), 185-193. DOI 10.14088/j.cnki.issn0439-8114.2019.22.043.

9. Wang, Y. L., Wang, L., Zhou, J. X., Hu, S. B., Chen, H. Z. et al. (2019). Research progress on heat stress of rice at flowering stage. Rice Science, 26(1), 1-10. DOI 10.1016/j.rsci.2018.06.009.

10. Li, J., Chen, G. P., Zhang, J. L., Shen, H., Kang, J. et al. (2020). Suppression of a hexokinase gene, SlHXK1, leads to accelerated leaf senescence and stunted plant growth in tomato. Plant Science, 298, 110544. DOI 10.1016/j. plantsci.2020.110544.

11. Wang, L., Ma, K. B., Lu, Z. G., Ren, S. X., Jiang, H. R. et al. (2020). Differential physiological, transcriptomic and metabolomic responses of arabidopsis leaves under prolonged warming and heat shock. BMC Plant Biology, 20(1), 1-15. DOI 10.1186/s12870-020-2292-y.

12. Zhong, Y. L., Xie, J. Y., Wen, S. Z., Wu, W. W., Tan, L. et al. (2020). TPST is involved in fructose regulation of primary root growth in arabidopsis thaliana. Plant Molecular Biology, 103(4-5), 511-525. DOI 10.1007/s11103020-01006-x.

13. Khan, A., Ahmad, M., Ahmed, M., Hussain, M. I. (2020). Rising atmospheric temperature impact on wheat and thermotolerance strategies. Plants (Basel), 10(1), E43. DOI 10.3390/plants10010043.

14. Escobar, M. R., Feussner, I., Valle, E. M. (2021). Mitochondrial small heat shock proteins are essential for normal growth of arabidopsis thaliana. Frontiers in Plant Science, 12, 600426. DOI 10.3389/fpls.2021.600426.

15. Avelange-Macherel, M. H., Rolland, A., Hinault, M. P., Tolleter, D., Macherel, D. (2019). The mitochondrial small heat shock protein HSP22 from Pea is a thermosoluble chaperone prone to co-precipitate with unfolding client proteins. International Journal of Molecular Sciences, 21(1), 97. DOI 10.3390/ijms21010097.

16. Li, K. P., Wong, C. H., Chen, C. C., Chen, S. S., Li, M. W. et al. (2021). GmDNJ1, a type-i heat shock protein 40 (HSP40), is responsible for both growth and heat tolerance in soybean. Plant Direct, 5(1), e00298. DOI 10.1002/ pld3.298.

17. Shi, J., Zhao, L., Zhu, Y. Q., Lou, X. P., Yu, J. Z. et al. (2018). Proteomic analysis of maize seedling leaves in response to heat stress. Acta Agriculturae Zhejiangensis, 30(6), 893-908. DOI 10.3969/j.issn.10041524.2018.06.03.

18. Haq, S., Khan, A., Ali, M., Khattak, A. M., Gai, W. X. et al. (2019). Heat shock proteins: Dynamic biomolecules to counter plant biotic and abiotic stresses. International Journal of Molecular Sciences, 20(21), 5321. DOI 10.3390/ ijms20215321.

19. Muthusamy, S. K., Dalal, M., Chinnusamy, V., Bansal, K. C. (2017). Genome-wide identification and analysis of biotic and abiotic stress regulation of small heat shock protein (HSP20) family genes in bread wheat. Journal of Plant Physiology, 211, 100-113. DOI 10.1016/j.jplph.2017.01.004. 